\title{
Efecto de la suplementación del diluyente sobre la calidad del semen de asno a la descongelación
}

\author{
Montoya, J.D. ' ; Rojano, B. ${ }^{2}$ y Restrepo, G. ${ }^{3 @}$
}

'Facultad de Ciencias Agrarias. Politécnico Colombiano Jaime Isaza Cadavid. Medellín. Colombia. ${ }^{2}$ Escuela de Química. Facultad de Ciencias. Universidad Nacional de Colombia. Medellín. Colombia.

${ }^{3}$ Departamento de Producción Animal.Facultad de Ciencias Agrarias. Universidad Nacional de Colombia. Medellín. Colombia.

Palabras Clave adicionales

Calidad seminal.

Crioprotectores.

Plasma seminal.

Yema de huevo.

Criopreservación.

\section{RESUMEN}

La congelación de semen de asno permite almacenar material genético por un periodo ilimitado de tiempo, por lo que puede ser empleado para generar bancos de germoplasma o para la cría de mulares y asnos. Esta investigación tuvo como objetivo evaluar alternativas de suplementación de un diluyente para la congelación de semen de asno. Se colectó el semen de cinco asnos criollos colombianos, dos eyaculados por animal $(n=10)$ y luego se congeló en diluyente Equiplus $®$ suplementado con $3 \%$ de glicerol (GLY) ó $5 \%$ de dimetilformamida (DMF), $2 \%$ ó $5 \%$ de yema de huevo centrifugada (YHC) y $10 \%$ ó $20 \%$ de plasma seminal (PS). Se realizó la evaluación post-descongelación de la calidad seminal y de la peroxidación lipídica (PL). Para el análisis estadístico se ajustaron modelos mixtos, se realizó un análisis de regresión y comparaciones de medias por la prueba de Tukey. El semen congelado con 5\% de DMF, 5\% de YHC y $10 \%$ de PS presentó valores superiores de movilidad, vitalidad e integridad de membrana $(p<0,05)$. La PL fue menor en diluyentes con $5 \%$ de YHC $(p<0,05)$. La suplementación del diluyente de congelación con una combinación de $5 \%$ de DMF, $5 \%$ de YHC y $10 \%$ de PS permite mejorar la calidad post-descongelación del semen de asno.

\section{Effect of extender supplementation on the post-thaw semen quality of donkey}

\section{SUMMARY}

Freezing of donkey semen allows storing genetic material for an unlimited period of time, which can be used to generate germoplasm banks or for breeding of mules or asses. This research aimed to evaluate supplementation alternatives for freezing donkey semen. Semen from five Colombian creole donkeys $(n=10)$ was collected and then frozen in Equiplus $\AA$ extender supplemented with $3 \%$ of glycerol (GLY) or $5 \%$ of dimethylformamide (DMF), $2 \%$ or $5 \%$ of centrifuged egg yolk (CEY) and $10 \%$ or $20 \%$ of seminal plasma (SP). Post-thaw evaluation of semen quality and lipid peroxidation (LP) was conducted. For statistical analysis mixed models were adjusted, regression analysis and comparison of means by the Tukey test were performed. Frozen semen with DMF, 5\% CEY and 10\% SP presented superior results of motility and membrane integrity $(p<0.05)$. The LP was lower in extenders with $5 \%$ CEY $(p<0.05)$. Supplementation of freezing extender with the combination of $5 \% \mathrm{DMF}, 5 \% \mathrm{CEY}$ and $10 \%$ SP allows to improve the post-thaw quality of asinine semen.

INFORMATION

Cronología del artículo.

Recibido/Received: 21.06.2016

Aceptado/Accepted: 14.02 .2017

On-line: 15.07.2017

Correspondencia a los autores/Contact e-mail:

grestreO@unal.edu.co

\section{INTRODUCCIÓN}

Son escasos los documentos de ingreso y difusión de la especie asnal en Colombia y se ignora cuándo se introdujo al país con fines de propagación, pero aparentemente esto ocurrió desde las primeras avanzadas de los españoles al igual que ocurrió con los caballos (Ramírez-Chaves et al., 2011). El semen de asno es uti- lizado en yeguas para la producción mular, dado que estos ejemplares son deseados en el medio rural (Canisso et al., 2008; Oliveira et al., 2014). Se han atribuido algunas ventajas al uso de semen descongelado de asno en programas de inseminación artificial, entre ellas evitar el rechazo de las yeguas a los garañones durante el cortejo y el apareamiento, así como superar las limitaciones físicas por el tamaño de los animales 
durante el servicio y facilitar el movimiento de los recursos genéticos (Madison et al., 2013). La congelación de semen de asno ha tenido un papel importante en la creación de bancos de germoplasma, que podrían ser utilizados en un futuro para reducir la depresión genética observada en las poblaciones de esta especie (Vilés-López, 2013).

La congelación del semen se relaciona con daños en la membrana plasmática, las mitocondrias y otras organelas de los espermatozoides (Ortiz et al, 2014; Darr et al., 2016). Esto se ve reflejado en la reducción de la movilidad, la viabilidad y la capacidad fecundante de dichas células (Najafi et al. 2014). Sin embargo, los espermatozoides de asno han demostrado ser resistentes a los procesos de refrigeración y criopreservación, al mantener características adecuadas de calidad seminal (Vilés-López, 2013).

Hasta la fecha, son escasas las investigaciones donde se aborda la congelación de semen asnal y es común que se adapten procesos empleados en equinos (Canisso et al., 2008; 2010). Los diluyentes para la congelación de semen requieren la adición de sustancias crioprotectoras, entre las cuales el glicerol (GLY) es posiblemente el crioprotector más empleado en la congelación del semen de équidos (Hoffmann et al., 2011; Rota et al., 2012). Sin embargo, se ha sugerido que la dosis tóxica de GLY en espermatozoides de asno parece ser casi la mitad que dicha dosis en espermatozoides equinos, además de comprometer la fertilidad del semen congelado (Vidament et al., 2009). Entre las principales alternativas, la dimetilformamida (DMF) es conocida por su efecto crioprotector en semen equino, que se atribuye a su menor toxicidad y su mayor capacidad de difusión a través de la membrana de los espermatozoides (Alvarenga et al., 2005), debido a su menor peso molecular. En un estudio reciente, se describió un efecto positivo de la DMF sobre la movilidad y la integridad de membrana de espermatozoides criopreservados de la raza asnal andaluza (Acha et al., 2015).

La adición de yema de huevo (YH) a los diluyentes para la congelación de semen, se debe a su aporte de lipoproteínas de baja densidad (LDL) compuestas por triglicéridos, fosfolípidos y colesterol (Moussa et al., 2002). Las LDL previenen la formación de cristales de hielo y protege la integridad de la membrana plasmática del choque térmico durante los procesos de congelación y descongelación (Hu et al., 2010; JianHong et al., 2011). Sin embargo, se ha descrito que la suplementación con $\mathrm{YH}$ genera riesgo microbiológico, alteraciones de la fosforilación oxidativa y la movilidad de los espermatozoides; así como produce complicaciones para realizar investigaciones bioquímicas, metabólicas y microscópicas del semen (Wall y Foote, 1999; Moussa et al., 2002). Debido a esto, se ha recurrido a la centrifugación de la $\mathrm{YH}$ con la finalidad de eliminar algunos de sus componentes y conservar principalmente las LDL, mediante la producción de yema de huevo centrifugada (YHC) y plasma de yema de huevo, las cuales han permitido mejorar la congelabilidad del semen equino (Pillet et al., 2011; Nouri et al., 2013).
Se conoce que el plasma seminal (PS) de los equinos contiene una gran diversidad de componentes involucrados en eventos como la activación de la movilidad espermática, la acción antimicrobiana, la neutralización de metabolitos, la capacitación espermática y la respuesta inflamatoria uterina postcoital (Kareskoski y Katila, 2008; Rodriguez-Martinez et al., 2011). Adicionalmente, en el PS se han identificado antioxidantes enzimáticos y no enzimáticos, los cuales tendrían la función de proteger los espermatozoides de los efectos nocivos del estrés oxidativo y se han asociado con la calidad seminal, la fertilidad y la respuesta a la congelación y descongelación del semen equino (Guasti et al., 2012; Waheed et al., 2013; Bucci et al., 2016). A pesar de lo anterior, poco se conoce de la composición, propiedades y efecto del plasma seminal en la congelación de semen de asnos (Rota et al., 2012).

Diferentes estudios demuestran que el semen congelado de asno presenta tasas de fertilidad bajas, cuando se utiliza para la inseminación de asnas en comparación con la inseminación de yeguas (Vidament et al., 2009; Oliveira et al., 2016). Lo cual podría deberse a una alta respuesta inflamatoria en el útero de las asnas por su interacción con el semen descongelado, provocando tasas de preñez muy bajas (Miró et al., 2013). La búsqueda de una combinación adecuada de suplementos para el diluyente de congelación, podría contribuir al mejoramiento de las condiciones de criopreservación del semen de asno; así como al aumento de su fertilidad en procesos de biotecnología reproductiva. El objetivo de este trabajo fue evaluar la suplementación de un diluyente para la congelación de semen, con diferentes crioprotectores (DMF y GLY) y proporciones de YHC (2\% y 5\%) y PS (10\% y $20 \%)$, con la finalidad de mejorar la calidad del semen de asno a la descongelación.

\section{MATERIALES Y MÉTODOS}

\section{ANIMALES Y COLECTA DE SEMEN}

Para la realización de este estudio se emplearon 5 asnos criollos colombianos (Equus asinus), localizados en el Valle del Aburra, Antioquia (Colombia), de edades comprendidas entre 4 y 8 años, condición corporal entre 7 y 8 (escala 1-9) (Warren, 2009) y con fertilidad comprobada. Bajo un régimen de una colecta semanal, se colectaron dos eyaculados por animal mediante vagina artificial (modelo Missouri, Minitube, Alemania), empleando una yegua como soporte. Los garañones se mantuvieron estabulados bajo condiciones similares de confinamiento, ejercicio y alimentación.

Una vez obtenido cada eyaculado, la fracción de gel se retiró por filtración usando un filtro de malla de nylon. Una alícuota de entre 5 y $10 \mathrm{ml}$ de semen se utilizó para la extracción de plasma seminal por centrifugación (850 x g durante 15 minutos). El resto del eyaculado se diluyó en proporción 1:1 (v:v) en EquiPlus $^{\circledR}$ (Ref. 13570/0202, Minitube, Alemania) a $37^{\circ} \mathrm{C}$ y se transportó en condiciones de refrigeración (Equitainer ${ }^{\circledR}$, Hamilton Research Inc, USA) al laboratorio de biotecnología animal del Politécnico Jaime Isaza Cadavid (Medellín, Colombia). Para cada muestra de semen fresco se evaluó el volumen mediante un tubo 
graduado y la concentración espermática mediante espectrofotometría (Spermacue ${ }^{\circledR}$, Minitube, Alemania). La movilidad espermática, la morfología anormal (MA), la vitalidad espermática (VE) y la integridad funcional de la membrana (HOS), se evaluaron mediante los métodos descritos más adelante. Solo se utilizaron para el estudio muestras con mínimo $60 \%$ de movilidad progresiva (MP) y una concentración mínima de $100 \times 10^{6}$ espermatozoides $/ \mathrm{mL}$.

\section{Criopreservación del SEMEN}

La criopreservación del semen se realizó mediante un protocolo modificado de congelación (Bustamante et al., 2009). El semen se dividió en 8 alícuotas de 10 $\mathrm{mL}$ de semen diluido cada una, las cuales se centrifugaron durante 12 minutos a $850 \times g$, se descartó el sobrenadante y se diluyeron aleatoriamente en el diluyente EquiPlus ${ }^{\circledR}$ (Ref. 13570/0202, Minitube, Alemania) suplementado con glicerol (GLY) al 3\% (SigmaAldrich, USA) o N,N-dimetilformamida (DMF) al 5\% (Sigma-Aldrich, USA), yema de huevo centrifugada (YHC) al 2\% ó 5\% y plasma seminal (PS) al 10\% ó 20\%, de la siguiente forma según el tratamiento:

\begin{tabular}{cccc} 
Tratamiento & Crioprotector & \% PS & $\%$ YHC \\
\hline 1 & GLY 3\% & 10 & 2 \\
2 & GLY 3\% & 20 & 2 \\
3 & GLY 3\% & 10 & 5 \\
4 & GLY 3\% & 20 & 5 \\
5 & DMF 5\% & 10 & 2 \\
6 & DMF 5\% & 20 & 2 \\
7 & DMF 5\% & 10 & 5 \\
8 & DMF 5\% & 20 & 5
\end{tabular}

La concentración de los crioprotectores se definió a partir de un ensayo previo de congelación de semen de asno (datos no publicados). La YHC se preparó de acuerdo a un protocolo modificado del descrito por Nouri et al. (2013). La YH se diluyó en proporción 3:1 (v:v) en agua ultra pura y se centrifugó a $1600 \mathrm{x}$ $g$ durante 100 minutos. El semen se mantuvo a $5^{\circ} \mathrm{C}$ durante 60 minutos y se envasó en pajuelas de $0,5 \mathrm{~mL}$ (IMV Technologies, Francia) a una concentración final de $100 \times 10^{6}$ espermatozoides/mL, en un equipo MRS1 Dual V2 (IMV Technologies, Francia). Las pajuelas se sometieron a vapores de nitrógeno líquido a una distancia de $4 \mathrm{~cm}$ de la superficie, durante 15 minutos y se almacenaron en un tanque con nitrógeno líquido. Tras un mes de almacenamiento, las pajuelas se descongelaron en agua a $37^{\circ} \mathrm{C}$ durante 1 minuto.

\section{EVALUACIÓN SEMINAL POST-DESCONGELACIÓN}

Se evaluó la movilidad y la cinética espermática mediante el sistema Sperm Class Analizer $\left(\mathrm{SCA}^{\circledR}{ }^{\circledR}, \mathrm{Mi}-\right.$ croptic S.L., España) de acuerdo a lo descrito por Restrepo et al. (2013). Se ajustó la configuración del sistema para la especie asnal, en base al tamaño de partícula, la concentración de la muestra y el número de espermatozoides evaluados. Se utilizó un microscopio de contraste de fase (Eclipse E200, Nikon, Inc., Japón) con una cámara digital (Scout SCA780, Basler, USA). Se evaluó la movilidad total (MT), movilidad progresiva (MP); las velocidades rectilínea (VSL), curvilínea (VCL) y media (VAP); los índices de linealidad (LIN), rectitud
(STR) y oscilación (IO): el desplazamiento lateral de la cabeza (ALH) y la frecuencia de batido (BCF).

Se avaluó la morfología anormal (MA) mediante la tinción con eosina-nigrosina (Barth y Oko, 1989). Sobre un portaobjetos se depositó una gota de muestra y una gota de eosina-nigrosina (Sigma-Aldrich, USA). Ambas gotas se mezclaron y se realizó un extendido, el cual se fijó sobre una platina térmica a $37^{\circ} \mathrm{C}$. Se realizó la evaluación de la morfología de 200 espermatozoides en un microscopio de contraste de fase (Eclipse E200, Nikon Inc., Japón).

Se determinó la vitalidad espermática (VE) utilizando el procedimiento descrito por Gamboa et al. (2010), con el kit Live/Dead (Molecular Probes Inc., USA). Se suspendieron $200 \mu \mathrm{L}$ de semen, en solución Hanks Heppes $(\mathrm{HH})$ con $1 \%$ de albúmina sérica bovina (BSA, Sigma Aldrich, USA), para una concentración de $20 \times 10^{6}$ espermatozoides / mL. Luego la mezcla se incubó a $37^{\circ} \mathrm{C}$ durante 8 minutos, con $6 \mathrm{mM}$ de SYBR14. Seguidamente se incubó de la misma manera, con $0,48 \mathrm{mM}$ de yoduro de propidio (IP). A continuación, a partir de una muestra de $5 \mu \mathrm{L}$, se realizó la evaluación de 200 espermatozoides, mediante un filtro UV-2A de un microscopio E200 con fluorescencia HBO (Nikon Inc., Japón).

Se avaluó la integridad funcional de la membrana plasmática (HOS) mediante la prueba hipoosmótica descrita por Neild et al. (1999). Se tomaron $100 \mu \mathrm{lde}$ semen y se adicionaron a un tubo con $500 \mu \mathrm{l}$ de una solución hipoosmótica de sacarosa 5,4\% (100 mOsmol / L). Esta mezcla se incubó a $38,5^{\circ} \mathrm{C}$ durante 30 minutos y seguidamente se evaluó la reacción de 200 espermatozoides por microscopia de contraste de fase (Eclipse E200, Nikon, Inc).

Se midió el nivel de peroxidación lipídica (PL) de la membrana plasmática de los espermatozoides de acuerdo al protocolo descrito por Ball y Vo (2002). Se emplearon $1000 \mu \mathrm{L}$ de semen descongelado, los cuales se centrifugaron a $600 \times g$ durante 10 minutos. El pellet con aproximadamente $100 \times 10^{6}$ espermatozoides, se suspendió en $1 \mathrm{~mL}$ de buffer fosfato de sodio (PBS, 75 $\mathrm{mM}, \mathrm{pH} 7,4$ ) con $10 \mu \mathrm{M}$ de $\mathrm{C}_{11}$-BODIPY $^{581 / 591}$ (Molecular Probes Inc., USA). Las muestras se dividieron en alícuotas de $300 \mu \mathrm{L}$, se sirvieron por triplicado en una microplaca de 96 pozos y se realizó la lectura cada 15 minutos (8 lecturas), de la fluorescencia roja y verde a longitudes de excitación y emisión (Ex / Em) de 590 $\mathrm{nm} / 635 \mathrm{~nm}$ y $485 \mathrm{~nm} / 535 \mathrm{~nm}$, respectivamente, en un espectrofluorímetro (LS-55 ${ }^{\circledR}$, Perkin Elmer, USA). Los resultados se generaron como unidades relativas de fluorescencia (URF). El grado de peroxidación lipídica de las células espermáticas se expresó como la relación entre la fluorescencia roja y verde (BODIPYrojo / BODIPY ${ }^{\text {verde }}$ ), donde BODIPY ${ }^{\text {rojo }}$ correspondió a la presencia de lípidos no peroxidados y la BODIPYverde correspondió a lípidos peroxidados.

\section{DISEÑO Y EVALUACIÓN ESTADÍSTICA}

Se realizó un diseño completamente al azar. Los resultados de calidad seminal se analizaron mediante el ajuste de modelos mixtos para cada variable dependiente. Para la evaluación de la peroxidación lipídica se realizó en modelo mixto de medidas repetidas en el tiempo y un análisis de regresión. En cada modelo se incluyeron los efectos fijos del asno (5), el eyaculado 
(10) y el tratamiento (8). Para asegurar la normalidad de los datos se utilizó prueba de Shapiro-Wilk y se realizaron transformaciones logarítmicas. La comparación de medias entre los tratamientos se realizó mediante la prueba de Tukey y se estableció un valor de $\mathrm{p}<0,05$. Todas las evaluaciones se realizaron con el programa SAS 9.2. (SAS Inst. Inc., USA).

\section{RESULTADOS Y DISCUSIÓN}

En el presente estudio, se encontraron para el semen fresco valores medios de volumen 45,7 $\pm 18,2 \mathrm{~mL}$, concentración $249 \pm 67 \times 10^{6}$ espermatozoides $/ \mathrm{mL}, \mathrm{MT}$ $92,2 \pm 7,1 \%$, MP $68,2 \pm 12,1 \%$, VCL 117,8 $\pm 19,5 \mu \mathrm{m} /$ seg, VSL 49,9 $\pm 8,8 \mu \mathrm{m} / \mathrm{seg}, \operatorname{VAP} 84.5 \pm 17.7 \mu \mathrm{m} / \mathrm{seg}$, MA $16.3 \pm 3.0 \%$, VE $88.2 \pm 6.5 \%$ y HOS $63.9 \pm 6.0 \%$, los cuales permiten catalogar las muestras seminales obtenidas como de buena calidad, de acuerdo a resultados reportados por otros autores en otras razas asnales (Canisso et al., 2010; Rota et al., 2012; Dorado et al., 2013).

En diferentes estudios con semen fresco de asnos, se ha encontrado una proporción de espermatozoides viables superior a la proporción de espermatozoides móviles (Miró et al., 2009; Miró et al., 2013), lo cual podría considerarse como congruente con lo esperado, toda vez que, la vitalidad como medida de la integridad de la membrana plasmática, está altamente relacionada con la funcionalidad de los espermatozoides. Sin embargo, en este estudio se encontró una ligera superioridad de MT respecto a VE. Carluccio et al. (2013), reportaron igualmente una ligera mayor proporción de espermatozoides móviles, respecto a aquellos con membrana integra. Esto podría ser atribuible a la variabilidad existente en los métodos de evaluación o en la configuración de los sistemas computarizados utilizados en las diferentes investigaciones.

Hasta la fecha los resultados de calidad post-descongelación del semen de asnos han sido variables (Contri et al., 2012; Rota et al., 2012). En la presente investigación, el semen congelado bajo los diferentes tra- tamientos, presentó mayor variabilidad para MT, MP, VE y HOS, con coeficientes de variación mayores al $40 \%$. Lo cual puede atribuirse no solo a las diferencias entre los tratamientos, sino también a la variación individual entre los reproductores, toda vez que los efectos fijos del asno, el eyaculado y el tratamiento fueron significativos para todas las variables dependientes de calidad seminal $(p<0,01)$. En équidos se ha descrito la existencia de una alta variabilidad entre machos en la crio-supervivencia del semen (Loomis y Graham, 2008), así como una marcada influencia de factores como la edad del reproductor (Dowsett y Knott, 1996), lo cual pudo tener una influencia marcada en este trabajo, dado el tamaño de la muestra. Todos los modelos estadísticos ajustados para las variables de calidad seminal fueron significativos $(\mathrm{p}<0,01)$. Los resultados de movilidad y cinética espermática para los diferentes tratamientos se presentan en la tabla I.

A excepción del tratamiento 6 , se observaron valores post-descongelación superiores de MT y MP para las muestras suplementadas con DMF (T5, T7, T8), respecto a aquellas suplementadas con GLY (T1 a T4) $(p<0,05)$. Estos resultados coinciden con lo encontrado por Acha et al. (2015), quienes describieron mejores resultados con DMF respecto al GLY (ambos suplementados al 2,5\%), para la movilidad y la integridad de membrana del semen criopreservado de asno andaluz. Gibb et al. (2013) atribuyeron los mejores resultados post-descongelación de la DMF, a la posible reducción del estrés osmótico ejercido en los espermatozoides, toda vez que su menor peso molecular favorece su paso más libre a través de la membrana. Adicionalmente, se conoce de la toxicidad que tiene el GLY en el semen de equinos y burros (Squires et al., 2004; Vidament et al., 2009).

Por otra parte, en las diferencias encontradas para los demás parámetros cinéticos del semen descongelado $(\mathrm{p}<0,05)$, no se observaron tendencias claras que pudiesen ser atribuibles a alguno de los suplementos empleados en el estudio (tabla I). Sin embargo, el T7 presentó menores valores de STR, LIN e IO, lo cual indicaría una interacción entre la combinación

Tabla I. Movilidad espermática post-descongelación de semen de asno (Post-thaw sperm motility of donkey semen).

\begin{tabular}{|c|c|c|c|c|c|c|c|c|}
\hline Parámetro & T1 & T2 & T3 & T4 & T5 & T6 & T7 & T8 \\
\hline MT (\%) & $22,8 \pm 13,9^{\text {cd }}$ & $18,1 \pm 20,2^{\mathrm{d}}$ & $28,9 \pm 17,1^{c}$ & $22,5 \pm 5,1^{c d}$ & $43,6 \pm 18,5^{b}$ & $23,7 \pm 16,7^{\mathrm{cd}}$ & $64,3 \pm 16,7^{a}$ & $49,4 \pm 18,9^{b}$ \\
\hline MP (\%) & $14,5 \pm 11,5^{\mathrm{cd}}$ & $11,7 \pm 17,1^{\mathrm{e}}$ & $19,5 \pm 13,5^{c}$ & $13,5 \pm 10,5^{\mathrm{de}}$ & $28,3 \pm 15,7^{b}$ & $14,5 \pm 17,2^{\mathrm{cd}}$ & $41,8 \pm 17,2^{\mathrm{a}}$ & $32,5 \pm 16,5^{b}$ \\
\hline VCL $(\mu \mathrm{m} / \mathrm{seg})$ & $79,9 \pm 16,4^{\mathrm{bc}}$ & $78,6 \pm 25,7^{c}$ & $87,7 \pm 21,5^{\mathrm{abc}}$ & $80,0 \pm 21,2^{\mathrm{bc}}$ & $89,7 \pm 16,2^{\mathrm{ab}}$ & $91,3 \pm 22,2^{\mathrm{a}}$ & $94,0 \pm 22,2^{\mathrm{a}}$ & $89,4 \pm 16,9^{\mathrm{ab}}$ \\
\hline VSL ( $\mu \mathrm{m} / \mathrm{seg})$ & $60,2 \pm 15,9^{\mathrm{ab}}$ & $57,1 \pm 21,6^{b}$ & $68 \pm 20,2^{a}$ & $59,7 \pm 19,9^{\text {ab }}$ & $63,7 \pm 15,2^{\mathrm{ab}}$ & $64,8 \pm 19,9^{a b}$ & $61,5 \pm 19,9^{a b}$ & $61,8 \pm 14,4^{\mathrm{ab}}$ \\
\hline VAP $(\mu \mathrm{m} / \mathrm{seg})$ & $69,7 \pm 16,9^{\mathrm{ab}}$ & $67,9 \pm 25,8^{b}$ & $77,8 \pm 21,6^{\mathrm{ab}}$ & $69,9 \pm 21,8^{\mathrm{ab}}$ & $77,3 \pm 16,9^{a}$ & $78,1 \pm 22,9^{a}$ & $79,0 \pm 22,9^{a}$ & $76,1 \pm 17,1^{\mathrm{ab}}$ \\
\hline LIN (\%) & $74,5 \pm 5,8^{\mathrm{cd}}$ & $71,1 \pm 11,7^{\mathrm{bc}}$ & $76,2 \pm 7,9^{a}$ & $73,3 \pm 7,6^{\mathrm{abc}}$ & $70,5 \pm 6,5^{\mathrm{bc}}$ & $70,2 \pm 7,4^{\mathrm{bc}}$ & $64 \pm 7,4^{d}$ & $68,8 \pm 5,7^{\mathrm{cd}}$ \\
\hline STR (\%) & $85,9 \pm 3,6^{a b}$ & $83,5 \pm 9,0^{\text {bcde }}$ & $86,6 \pm 5,2^{a}$ & $84,9 \pm 4,8^{\text {cde }}$ & $82,1 \pm 4,4^{\mathrm{cd}}$ & $82,7 \pm 5,0^{\mathrm{bcd}}$ & $77,3 \pm 5,0^{\mathrm{e}}$ & $81,1 \pm 4,1^{d}$ \\
\hline $10(\%)$ & $86,6 \pm 4,0^{\mathrm{ab}}$ & $84,5 \pm 7,8^{\mathrm{bc}}$ & $87,7 \pm 4,9^{a}$ & $86,2 \pm 5,4^{\mathrm{ab}}$ & $85,7 \pm 4,2^{\mathrm{abc}}$ & $84,7 \pm 5,3^{\text {bc }}$ & $83,1 \pm 5,3^{c}$ & $84,7 \pm 3,9^{b c}$ \\
\hline ALH $(\mu \mathrm{m})$ & $2,2 \pm 0,2^{\mathrm{d}}$ & $2,3 \pm 0,5^{c d}$ & $2,2 \pm 0,3^{d}$ & $2,3 \pm 0,3^{c d}$ & $2,5 \pm 0,3^{\mathrm{bc}}$ & $2,8 \pm 0,3^{a}$ & $2,7 \pm 0,3^{\mathrm{ab}}$ & $2,6 \pm 0,2^{b}$ \\
\hline $\mathrm{BCF}(\mathrm{Hz})$ & $8,8 \pm 0,9^{a b}$ & $8,1 \pm 1,7^{c}$ & $8,9 \pm 1,6^{a}$ & $8,8 \pm 0,9^{a b}$ & $8,2 \pm 0,6^{b c}$ & $8,5 \pm 0,5^{\mathrm{abc}}$ & $7,9 \pm 0,5^{c}$ & $8,2 \pm 0,7^{\mathrm{bc}}$ \\
\hline
\end{tabular}

MT: movilidad total. MP: movilidad progresiva. VSL: velocidad rectilínea. VCL: velocidad curvilínea. VAP: velocidad media. LIN: índice de linealidad. STR: índice de rectitud. IO: índice de oscilación. ALH: desplazamiento lateral de la cabeza. BCF: frecuencia de batido. T1: GLY, PS $10 \%$, YHC 2\%; T2: GLY, PS $20 \%$, YHC $2 \%$; T3: GLY, PS $10 \%$, YHC 5\%; T4: GLY, PS 20\%, YHC 5\%; T5: DMF, PS $10 \%$, YHC 2\%; T6: DMF, PS 20\%, YHC 2\%; T7: DMF, PS 10\%, YHC 5\% y T8: DMF, PS 20\%, YHC 5\%. Los resultados se expresan como media \pm desviación estándar. Letras diferentes denotan diferencia estadística significativa $(p<0,05)$. 
de suplementos de dicho tratamiento y la cinética espermática post-descongelación. Madison et al. (2013) hallaron para la mayoría de parámetros cinéticos valorados a la descongelación valores superiores en las muestras seminales de asnos suplementadas con una baja proporción de YH (5\% vs. 20\%). Mientras Nouri et al. (2013) encontraron que la suplementación del diluyente con lecitina de soya en combinación con YHC al $2 \%$, tiene un efecto favorable sobre MT y MP, y con YHC al 4\% sobre VSL y LIN. Las LDL presentes en la YHC se adhieren a la membrana plasmática, dotando al espermatozoide de una mayor protección contra los cambios de temperatura durante la congelación (Morillo et al., 2012).

La adición de PS a los diluyentes para la congelación del semen, se ha evaluado como una alternativa para el mejoramiento de su calidad post-descongelación, a través de la reducción del estrés oxidativo (Guasti et al., 2012; Waheed et al., 2013). Incluso resultados previos en semen de caballos criollos colombianos, mostraron una reducción de la peroxidación lipídica en el semen descongelado, por la adición de PS al 10\% y 20\% al diluyente de congelación (Pizarro et al., 2013). En asnos se ha descrito la adición de PS para la redilución de semen descongelado antes de la inseminación artificial, con resultados que permitieron evidenciar una tendencia hacia el mejoramiento de la fertilidad (Rota et al., 2012). En esta investigación se observó que los tratamientos con 10\% de PS (T1, T3, T5 y T7) presentaron en la mayoría de los casos mejores resultados de MT y MP, que sus tratamientos equivalentes con $20 \%$ de PS (T2, T4, T6 y T8) $(\mathrm{p}<0,05)$. Otros investigadores han registrado un efecto deletéreo del PS en la congelación de semen equino, cuando se su- plementa en proporciones iguales o superiores al 20\% (Alghamdi et al., 2002; Moore et al., 2005). Sin embargo, la calidad post-descongelación del semen también depende de la interacción entre los suplementos empleados en el diluyente, dado que con el uso de DMF y $20 \%$ de PS se hallaron resultados superiores para MT y MP (tabla I), así como para MA, VE y HOS (figura 1), cuando se adicionó un 5\% de YHC (T8) en lugar de un $2 \%$ de este suplemento (T6) $(\mathrm{p}<0,05)$. De otro lado, no se observó este mismo resultado cuando se utilizó GLY como crioprotector permeable y 20\% de PS (T2 y T4). Lo cual muestra que el efecto del PS está de cierta forma condicionado por otros componentes del diluyente y al parecer con una particular interacción con la YH. Algunos estudios han descrito un efecto positivo sobre la calidad seminal por efecto de la interacción entre el PS y la YH (Ferreira et al., 2014).

El uso de DMF incluso con diferentes proporciones de PS y YHC permitió resultados superiores de movilidad espermática (tabla I), vitalidad e integridad de la membrana plasmática $(\mathrm{p}<0,05)$ (figura 1). Para la MA no se observó un efecto fácilmente atribuible a alguno de los suplementos o tratamientos, a pesar de que la menor proporción de espematozoides anormales se presentó para el T6 ( $\mathrm{p}<0,05)$ (figura 1). El T7 presentó resultados superiores de calidad seminal representados en la MT, MP (tabla I), VE y HOS (figura 1) del semen descongelado $(\mathrm{p}<0,05)$. Otros autores han obtenido resultados que podrían estar igualmente determinados por la combinación de diferentes suplementos en el diluyente de congelación. Nouri et al. (2013) encontraron que la suplementación del diluyente con lecitina de soya en combinación con YHC al $2 \%$, mejora la integridad de membrana (HOS) y reduce

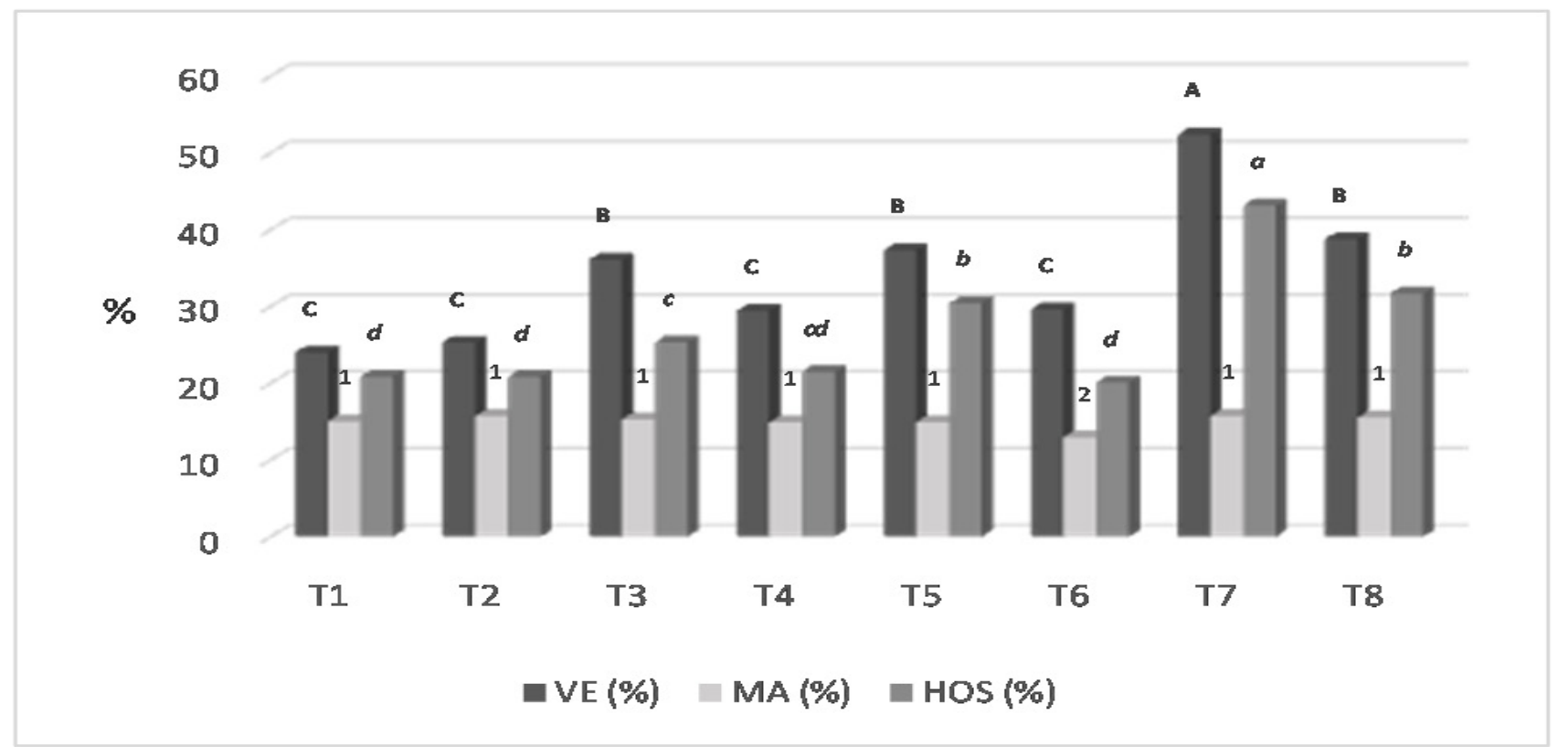

Figura 1. Morfología, vitalidad espermática e integridad de membrana post-descongelación de semen de asno (Post-thaw sperm vitality, morphology and membrane integrity of donkey semen).

MA: Morfología anormal.VE: vitalidad espermática. HOS: Integridad funcional de membrana.

T1: GLY, PS 10\%, YHC 2\%; T2: GLY, PS 20\%, YHC 2\%; T3: GLY, PS 10\%, YHC 5\%; T4: GLY, PS 20\%, YHC 5\%; T5: DMF, PS 10\%, YHC 2\%; T6: DMF, PS 20\%, YHC 2\%; T7: DMF, PS 10\%, YHC 5\% y T8: DMF, PS 20\%, YHC 5\%. Se denota diferencia estadística significativa $(\mathrm{p}<0,05)$ con letras mayúsculas para VE $(\mathrm{A}, \mathrm{B}, \mathrm{C})$, números arábigos para MA $(1,2)$ y letras minúsculas para $\operatorname{HOS}(a, b, c, d)$. 


\begin{tabular}{lcccc}
\hline \multicolumn{4}{l}{ Tabla II. Peroxidación lipídica post-descongelación de semen de asno (Post-thaw lipid peroxidation of donkey semen). } \\
\hline \multirow{2}{*}{ Tratamiento } & $\begin{array}{c}\text { Peroxidación } \\
\text { BODIPYrjo / BODIPYverde }\end{array}$ & Intercepto $\beta_{0}$ & Análisis de regresión \\
\cline { 3 - 5 } T1 & $3,63 \pm 0,89^{\mathrm{c}}$ & 3,40 & Tiempo $\beta_{1}$ & 0,0031 \\
T2 & $4,03 \pm 1,57^{\mathrm{b}}$ & 3,58 & 0,0064 & 0,566 \\
T3 & $4,82 \pm 1,20^{\mathrm{a}}$ & 4,44 & 0,0054 & 0,031 \\
T4 & $5,06 \pm 1,56^{\mathrm{a}}$ & 4,57 & 0,0069 & 0,035 \\
T5 & $3,48 \pm 0,88^{\mathrm{c}}$ & 3,10 & 0,0053 & 0,002 \\
T6 & $4,15 \pm 1,31^{\mathrm{b}}$ & 3,66 & 0,0069 & 0,012 \\
T7 & $4,91 \pm 1,12^{\mathrm{a}}$ & 4,38 & 0,0075 & 0,001 \\
T8 & $4,79 \pm 1,19^{\mathrm{a}}$ & 4,24 & 0,0077 & 0,002 \\
\hline
\end{tabular}

T1: GLY, PS 10\%, YHC 2\%; T2: GLY, PS 20\%, YHC 2\%; T3: GLY, PS 10\%, YHC 5\%; T4: GLY, PS 20\%, YHC 5\%; T5: DMF, PS 10\%, YHC $2 \%$; T6: DMF, PS $20 \%$, YHC $2 \%$; T7: DMF, PS $10 \%$, YHC $5 \%$ y T8: DMF, PS $20 \%$, YHC $5 \%$.

Los resultados de peroxidación se expresan como media \pm desviación estándar. Letras diferentes denotan diferencia estadística significativa $(p<0,05)$. $\beta 1$ : Incremento de la relación BODIPYrojo / BODIPYverde por minuto.

la MA, mientras se observó un efecto deletéreo de la YHC al $4 \%$ para estos mismos parámetros. Jepsen et al. (2010) reportaron diferencias en los resultados de calidad seminal y tasas de gestación, a partir de semen de asno congelado con diferentes combinaciones de GLY, $\beta$-ciclodextrina y YH (5\% vs. 20\%).

La peroxidación lipídica es considerada como un indicador del estrés oxidativo generado durante la congelación del semen. Se ha descrito que un incremento en la producción de especies reactivas de oxigeno (ERO) produce la peroxidación de los ácidos grasos poliinsaturados en la membrana plasmática de los espermatozoides equinos (García et al., 2011). Esto genera daños subletales en los espermatozoides que involucran el descenso de la movilidad, pérdida de fluidez de la membrana y reacción acrosómica anormal, lo cual termina por comprometer la fertilidad (Ortega et al., 2009; Vasconcelos et al., 2013). El modelo estadístico ajustado para la evaluación de la peroxidación lipídica fue significativo, así como los efectos fijos del asno, el eyaculado y el tratamiento $(p<0,001)$. Se encontró una media para la relación de peroxidación

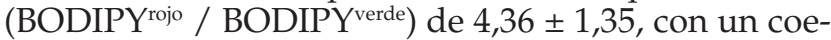
ficiente de variación de $17,6 \%$. Se observó una mayor relación de peroxidación lipídica (BODIPYrojo / BODIPYverde) para los tratamientos con mayor proporción de YHC (T3, T4, T7 y T8) (tabla II). De igual forma, el análisis de regresión arrojó para los mismos tratamientos, coeficientes de regresión mayores y significativos para el incremento en la relación BODIPY rojo / BODIPYverde en el tiempo $(\mathrm{p}<0,05)$ (tabla II). Ambos análisis indicarían que la YHC tuvo un efecto de reducción de la peroxidación lipídica cuando se suplemento en mayor proporción (5\%), tanto en muestras congeladas en presencia de GLY o DMF y diferentes proporciones de PS. Lo cual, a excepción del T5, coincidió con aquellos tratamientos que presentaron mejores resultados de calidad seminal. Este efecto podría ser en parte atribuido a las LDL y los fosfolípidos presentes en la YHC, dado que se ha observado un efecto protector de éstos sobre la movilidad y la integridad de membrana plasmática durante la criopreservación del semen (Vera-Munoz et al., 2011; Pillet et al., 2012). Sin embargo, los mecanismos precisos por los cuales las LDL protegen los espermatozoides durante la congelación aún no están claros; a pesar de esto, se han propuesto como hipótesis diferentes formas de interacción de las LDL y sus componentes con la membrana plasmática (Pillet et al., 2012).

Con los resultados de este estudio, se hace evidente que el uso de la combinación de suplementos (DMF 5\%, YHC 5\% y PS 10\%) para la congelación de semen de asnos criollos colombianos, contribuye al mejoramiento de diferentes parámetros de calidad seminal in vitro, así como a la reducción de alteraciones espermáticas, tales como la peroxidación lipídica. Sin embargo, diferentes estudios han demostrado que a pesar de contar con semen criopreservado de excelente calidad, su fertilidad en la inseminación de asnas es reducida (Oliveira et al., 2016). Lo que ha llevado a la búsqueda de alternativas que permitan mejorar la respuesta uterina y las estrategias para la inseminación con semen criopreservado (Jepsen et al., 2010; Rossi et al., 2012; Miró et al., 2013). Por lo anterior, y a partir de los resultados de esta investigación, se plantea la necesidad de evaluar la contribución de los suplementos empleados en el semen criopreservado mediante pruebas de fertilidad in vivo.

\section{CONCLUSIONES}

La suplementación del diluyente utilizado para la congelación de semen de asnos criollos colombianos con la combinación de DMF al 5\%, YHC al 5\% y PS al $10 \%$ permite mejorar la calidad espermática a la descongelación. El uso de PS y YHC como alternativas para la congelación de semen de asnos permiten mejorar la movilidad, la vitalidad y la integridad de membrana de los espermatozoides, así como reducir los niveles de peroxidación lipídica post-descongelación.

\section{AGRADECIMIENTOS}

A la Dirección de Investigación y Posgrados del Politécnico Colombiano Jaime Isaza Cadavid por la financiación de la investigación. 


\section{BIBLIOGRAFÍA}

Acha, D.;Hidalgo, M.; Ortiz, I., Galvez,M.; Carrasco, J.; Gomez-Arrones, V. and Dorado, J. 2015. Freeze ability of Andalusian donkey (Equus asinus) spermatozoa: effect of extenders and permeating cryoprotectants. Reprod Fert Develop, 28: 1990-1998.

Alghamdi, A.; Troedsson, M.; XUE, J. and Crabo, B. 2002. Effect of seminal plasma concentration and various extenders on post-thaw motility and glass wool-Sephadex filtration of cryopreserved stallion semen. Am J Vet Res, 63: 880-885.

Alvarenga, M.; Papa, F.; Landim-Alvarenga, F. and Medeiros, A. 2005. Amides of cryoprotectants for freezing stallion semen: a review. Anim Reprod Sci, 89: 105-113.

Ball, B. and VO, A. 2002. Detection of lipid peroxidation in equine spermatozoa based upon the lipophilic flourescent dye $C_{11}-$ BODIPY $^{581 / 591}$. J Androl, 23: 259-263.

Barth, A. and Oko, R. 1989. Abnormal morphology of bovine spermatozoa. 1 ed. lowa State University Press. lowa, U.S.A. 285 pp.

Bucci, D.; Giaretta, E.; Spinaci, M.; Rizzato, G.; Isani, G.; Mislei, B.; Mari, G.; Tamanini, C. and Galeati, G. 2016. Characterization of alkaline phosphatase activity in seminal plasma and in fresh and frozen-thawed stallion spermatozoa. Theriogenology, 15: 288-295.

Bustamante, I.; Pederzolli, C.; Sgaravatti, A.; Gregory, R.; Dutra, C.; Jobim, M. and Mattos, R. 2009. Skim milk-egg yolk based semen extender compensates for non-enzymatic antioxidant activity loss during equine semen cryopreservation. Anim Reprod, 6: 392-399.

Canisso, I.; Carvalho, G.; Davies-Morel, M.; Guimarães, J. and McDonnell, S. 2010. Sexual behavior and ejaculate characteristics in Pêga donkeys (Equus asinus) mounting estrous horse mares (Equus caballus). Theriogenology, 73: 56-63.

Canisso, I.; Souza, F.; Escobar, J.; Ribeiro, G.; Davies-Morel, M.; Capistrano da Silva, E., Guimarães J. and Linhares-Lima, A. 2008. Congelamiento de semen de burro (Equus asinus). Rev Investig Vet Perú, 19: 113-125.

Carluccio, A; Panzani, S; Contri, A; Bronzo, V; Robbe, D. and Veronesi, M. 2013. Influence of season on testicular morphometry and semen characteristics in Martina Franca jackasses. Theriogenology, 79: 502-507.

Contri, A.; Alessia, G.; Domenico, R.; Michele, P.; Sfirro, M. and Carluccio, A. 2012. Effect of sperm concentration on characteristics of frozen-thawed semen in donkeys. Anim Reprod Sci, 136: 74-80.

Darr, C.; Cortopassi, G.; Datta, S.; Varner, D. and Meyers, S. 2016. Mitochondrial oxygen consumption is a unique indicator of stallion spermatozoal health and varies with cryopreservation media. Theriogenology, 86: 1382-1392.

Dorado, J.; Acha, D.; Gálvez, M.; Ortiz, I.; Carrasco, J.; Díaz, B.; Gómez-Arrones, V.; Calero-Carretero, R. And Hidalgo, M. 2013. Sperm motility patterns in Andalusian donkey (Equus asinus) semen: Effects of body weight, age, and semen quality. Theriogenology, 79: 1100-1 109.

Dowsett, K. and Knott, L. 1996. The influence of age and breed on stallion semen. Theriogenology, 46: 397-412.

Ferreira, V.; Bougr de Mello, Marco.; Moreira da Fonseca, C.; Ferreira, A.; Cardoso, J.; Silva, R. and Martins, W. 2014. Effect of seminal plasma and egg yolk concentration on freezability of goat semen. Rev Bras Zootecn, 43: 513-518.

Gamboa, S.; Rodrigues, A.; Henriques, L.; Batista, C. and Ramalho-Santos, J. 2010. Seasonal functional relevance of sperm characteristics in equine spermatozoa. Theriogenology, 73: 950-958.

García, B.; Fernández, L.; Ferrusola, C.; Salazar-Sandoval, C.; Rodríguez, A.; Martinez, H.; Tapia, J.; Morcuende, D. and Peña, F. 2011. Membrane lipids of the stallion spermatozoon in relation to sperm quality and susceptibility to lipid peroxidation. Reprod Domest Anim, 46: 141-148.

Gibb, Z.; Morris, L.; Maxwell, W. and Grupen, C. 2013. Dimethyl formamide improves the postthaw characteristics of sex-sorted and nonsorted stallion sperm. Theriogenology, 79: 1027-1033.
Guasti, P.N.; Monteiro, G.A. and Papa, F.O. 2012. Componentes do plasma seminal e sua influência sobre a criopreservação e fertilidade de espermatozoides equinos. Vet Zootec, 19: 169-180.

Hoffmann, N.; Ondelholf, H.; Morandini, C.; Rohn, K. and Sieme, H. 2011. Optimal concentration of cryoprotectant agent for freezing stallion that are classified "good" or "poor" for freezing. Anim Reprod Sci, 125: 112-118.

Hu, J.; Li, Q.; Zan, L.; Jiang, Z.; An, J.; Wang, L. and Jia, Y. 2010. The cryoprotective effect oflow-densitylipoproteins in extenders on bull spermatozoa following freezing-thawing. Anim Reprod Sci, 117: 11-17.

Jepsen, R.; Evans, L. and Youngs, C. 2010. Use of direct thaw insemination to establish pregnancies with frozen-thawed semen from standar Jack. J Equine Vet Sci, 30: 651-656.

Jian-Hong, H.; Zhong-Liang, J.; Rui-Kai, L.; Qing-Wang, L.; Shu-Shan, Z.; Lin-Sen, Z.; Yao-Kun, L. and Xin, L. 2011. The advantages of low-density lipoproteins in the cryopreservation of bull semen. Cryobiology, 62: 83-87.

Kareskoski, M. and Katila, T. 2008. Components of stallion seminal plas$\mathrm{ma}$ and effects of seminal plasma on sperm longevity. Anim Reprod Sci, 107: 249-256.

Loomis, P. and Graham, J. 2008. Commercial semen freezing: Individual male variation in cryosurvival and the response of stallion sperm to customized freezing protocols. Anim Reprod Sci, 105: 119-128.

Madison, J.; Evans, L. and Youngs, C. 2013. The effect of 2-hydroxypropylb-cyclodextrin on post-thaw parameters of cryopreserved jack and stallion semen. J Equine Vet Sci, 33: 272-278.

Miró, J; Taberner, E; Rivera, M; Peña, A; Medrano, A; Rigau, T. and Peñalba, A. 2009. Effects of dilution and centrifugation on the survival of spermatozoa and the structure of motile sperm cell subpopulations in refrigerated Catalonian donkey semen. Theriogenology, 72: 1017-1022.

Miró, J.;Vilés, K.;García, W.; Jordana, J. and Yeste, M. 2013. Effect of donkey seminal on sperm-polymorphonuclear neutrophils attachment in vitro. Anim Reprod Sci, 140: 164-178.

Moore, A.; Squires, E. and Graham, J. 2005. Effect of seminal plasma on the cryopreservation of equine spermatozoa. Theriogenology, 63: 2372-2381.

Morillo, A; Balao da Silva, C.; Macías-García, B.; Gallardo, J.; Tapia, J. and Aparicio, I. 2012. Dimethyformamide improves in vitro characteristics of thawed stallion sparmatozoa reducing sublethal damage. Reprod Domest Anim, 47: 995-1002.

Moussa, M.; Martinet, V.; Trimeche, A.; Tainturier, D. and Anton, M. 2002. Low density lipoproteins extracted from hen egg yolk by an easy method: cryoprotective effect on frozen-thawed bull semen. Theriogenology, 57: 1695-1706.

Najafi, A.; Daghigh, H.; Mohammadi, H.; Najafi, H.; Zanganeh, Z.; Sharafi, M.; Martinez, F. and Adeldust, H. 2014. Different concentrations of cysteamine and ergothioneine improve microscopic and oxidative parameters in ram semen frozen with a soybean lecithin extender. Cryobiology, 69: 68-73.

Neild, D.; Chaves, G.; Flores, M.; Mora, N.; Beconi, M.; Agiiero, A. 1999. Hypoosmotic test in equine spermatozoa. Theriogenology, 51: 721-727.

Nouri, H.; Towwhidi, A.; Zhandi, M. and Sadeghi, R. 2013. The effects of centrifuged egg yolk used with INRA plues soybean lecithin extender on semen quality to freeze Caspian horse semen. J Equine Vet Sci, 33: 1050-1053.

Oliveira, R.; Rates, D.; Pugliesi, G.; Ker, P.; Arruda, R.; Moraes, E. and Carvalho, G. 2014. Use of Cholesterol-loaded cyclodextrin in donkey semen cryopreservation improves sperm viablity but results in low fertility in mares. Reprod Domest Anim, 49: 845-850.

Oliveira, J; Oliveira, P; Melo, C; Guasti, P; Monteiro, G; Sancler da Silva, Y; Papa, P; Alvarenga, M; Dell'Aqua, J. and Papa F. 2016. Strategies to improve the fertility of fresh and frozen donkey semen. Theriogenology, 85: 1267-1273.

Ortega, C.; González, L.; Morrell, J.; Salazar, C.; Macías, B.; RodríguezMartínez, H, Tapia, J. and Peña, F. 2009. Lipid peroxidation, assessed 
with BODIPY-C1 1 , increases after cryopreservation of stallion spermatozoa, is stallion-dependent and is related to apoptotic-like changes. Reproduction, 138: 55-63.

Ortiz, I.; Dorado, J.; Ramírez, L.; Morrell, J.; Acha, D.; Urbano, M.; Gálvez, M.; Carrasco, J.; Gómez-Arrones, V.; Calero-Carretero, R. and Hidalgo M. 2014. Effect of single layer centrifugation using Androcoll-E-Large on the sperm quality parameters of cooled-stored donkey semen doses. Animal, 8: 308-315.

Pillet, E.; Duchamp, G.; Batellier, F.; Beaumal, V.; Anton, M.; Desherces, S.; Schmitt, E. and Magistrini, M. 2011 . Egg yolk plasma can replace egg yolk in stallion freezing extenders. Theriogenology, 75: 105-114.

Pillet, E.; Labbe, C.; Batellier, F.; Duchamp, G.; Beaumal, V.; Anton, M.; Desherces, S.; Schmitt, E. and Magistrini, M. 2012. Liposomes as an alternative to egg yolk in stallion freezing extender. Theriogenology, 77: 268-279.

Pizarro, E.; Restrepo, G.; Echeverry, J. y Rojano, B. 2013. Efecto del plasma seminal sobre el estado redox del semen equino criopreservado. Rev MVZ Córdoba, 18: 3672-3680.

Ramírez-Chaves, H.E.; Ortega-Rincón, M.; Pérez, W.y Martin, J. Historia de mamíferos exóticos en Colombia. Boletín Científico Centro de Museos, Museo de Historia Natural, 15: 139-152.

Restrepo, G.; Ocampo, D. y Velásquez, A. 2013. Evaluación de la movilidad del semen criopreservado de caballos criollo colombiano por un sistema analizador de clase. Rev UDCA Act \& Div Cient, 16: 445-450.

Rodriguez-Martinez, H.; Kvist, U.; Ernerudh, J.; Sanz, L. and Calvete, J. 2011 . Seminal plasma proteins: what role do they play?. Ame J Reprod Immunol, 66: 11-22.

Rossi R, Filho J, Palhares M, Ribeiro E, Resende Y, Anjos F. 2012. Efeito do número de inseminações artificiais sobre a fertilidade de éguas inseminadas com sêmen asinino diluído e resfriado a $5^{\circ} \mathrm{C}$ por 12 horas de armazenamento. Arq Bras Med Vet Zootec, 64: 114-119.

Rota, A.; Panzini, D.; Sabatini, C. and Camillo, F. 2012. Donkey jack (Equus asinus) semen cryopreservation: Studies of seminal parameters, post breeding inflammatory response, and fertility in donkey jennies. Theriogenology, 78: 1846-1854.
Squires, E.; Keith, S. and Graham, J. 2004. Evaluation of alternative cryoprotectants for preserving stallion spermatozoa. Theriogenology, 62: 1056-1065.

Vasconcelos, J.; Chaveiro, A.; Góis, A. and Moreira da Silva F. 2013. Effects of a-tocopherol and ascorbic acid on equine semen quality after cryopreservation. J Equine Vet Sci, 33: 787-793.

Vera-Munoz, O.; Amirat-Briand, L.; Bencharif, D.; Anton, M.; Desherces, S.; Shmitt, E.; Thorin, C. and Tainturier, D. 2011 . Effect of low-density lipoproteins, spermatozoa concentration and glycerol on functional and motility parameters of bull spermatozoa during storage at $4{ }^{\circ} \mathrm{C}$. Asian J Androl, 13: 281-286.

Vidament, M.; Vincent, P.; Martin, F.; Magistrini, M. and Blesbois, E. 2009. Differences in ability of jennies and mares to conceive with cooled and frozen semen containing glycerol or not. Anim Reprod Sci, 112: 22-35.

Vilés-López, K. 2013. Estudio de la inflamación endometrial post inseminación con semen congelado en la burra catalana y posibilidades de control. Tesis doctoral. Facultad de Veterinaria. Universidad Autónoma de Barcelona. España.

Waheed, M.; El-Bahr, S. and Al-Haider, A. 2013. Influence of seminal plasma antioxidants and osteopontin on fertility of the arabian horse. J Equine Vet Sci, 33: 705-709.

Wall, R. and Foote, R. 1999. Fertility of bull semen frozen and store in clarified egg yolk-Tris-glycerol extender. J Dairy Sci, 82: 817-821.

Warren, L. 2009. Feeding the stallion. Alberta Agriculture and Rural Development. Alberta. USA. 